\title{
Computer Aided Design of Grinding Wheel for Drill Flute Production
}

\author{
Janko Jovanović ${ }^{1}$, Obrad Spaić ${ }^{2}$, Radoslav Tomović ${ }^{1}$ and Rade Ivanković ${ }^{2}$ \\ 1. Faculty of Mechanical Engineering, University of Montenegro, Podgorica 81000, Montenegro \\ 2. Production and Management Faculty, East Sarajevo University, Trebinje 89101, Republika Srpska
}

Received: June 12, 2014 / Accepted: June 25, 2014 / Published: September 25, 2014.

\begin{abstract}
Twist drill flute profile design is necessary in order to determine the required grinding wheel profile for a flute production. An accurate drill flute profile design is generated for two-flute conical twist drills using analytical equations to generate a drill flute profile design needed for the production of twist drills with straight lips. The required grinding wheel profile for a flute production was expressed in digitized form as well as in terms of two curve-fitted circular arcs. The drill flute profile geometry can never be precisely generated when required grinding wheel profile is represented by two circular arcs and the generated flute profile is just a very good approximation of the design flute profile. A CAD (computer aided design) software has been developed using MATLAB to determine the required grinding wheel profile for generating a given drill flute profile design.
\end{abstract}

Key words: Grinding wheel profile, drill flute profile, twist drill, CAD.

\section{Introduction}

The drill manufacturing primarily consists of two grinding steps, namely, grinding the flute faces and grinding the flank faces. The parameters of these grinding operations determine the geometric parameters of the drill. The parameters such as point angle and web thickness are the implicit functions of the drill's manufacturing parameters.

During the flute grinding, the grinding wheel rotates in-place with the drill simultaneously rotating about and moving down its axis. The dual motion of the drill controls the helix angle of the flute and the position and profile of the grinding wheel controls the cross-section of the drill flute. In a two-flute drill, this is performed twice at orthogonal positions to generate both flutes. During the flank grinding, the grinding wheel rotates about a fixed axis to form the grinding cone of a cone semi-angle $\theta$, as shown in Fig. 1, and the drill rotates

Corresponding author: Janko Jovanović, Ph.D., associate professor, research fields: computer aided design, computer aided engineering and computer aided manufacturing. E-mail: janko@ac.me. about the axis of grinding cone. This grinding is also performed twice from symmetric positions to generate both flank surfaces. These flank surfaces can be considered as the sections of the grinding cones.

Galloway [1] initiated a formal study of a drill geometry in his paper where he discussed several aspects of the drilling process and gave the sophisticated analysis of a drill point sharpening based on a conical grinding concept for the straight lipped drills shown in Fig. 1. The equations for the relevant part of a flute geometry required to give straight lips were derived together with the expressions for a lip clearance and rake angles along the drill lips. Galloway [1] pointed out that the flank surface of the drill would be fully determined if the semi-cone angle $\theta$ and the cone apex position with respect to the drill axis denoted by parameters $d$ and $s$ in Fig. 1 were known in addition to the specified drill point angle $2 p$, helix angle $\delta$, web thickness $2 W$ and diameter $D$.

The subsequent researchers built on Galloway's [1] basic framework and extended his analytical equations to develop computer-based models. Fuji and DeVries 


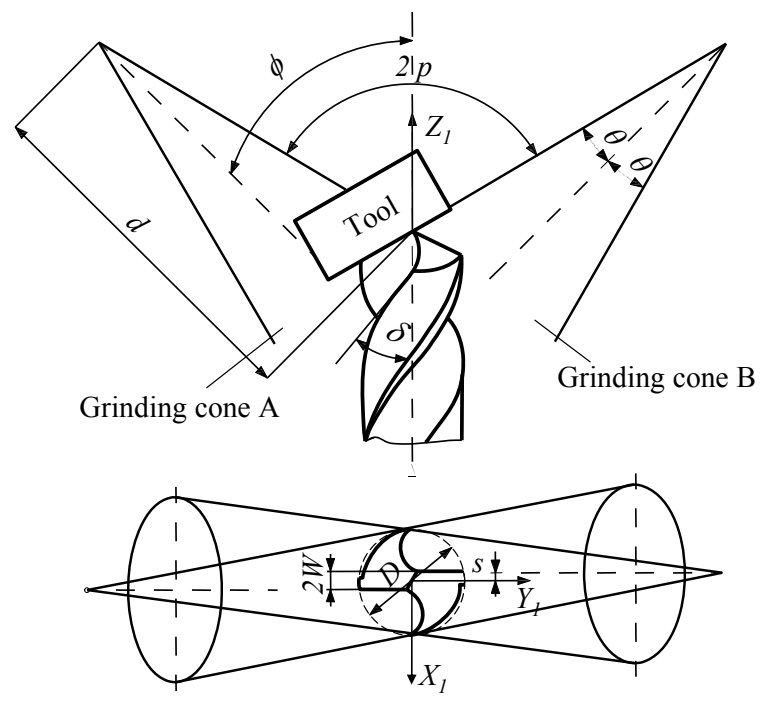

Fig. 1 Drill point sharpening based on a conical grinding concept.

$[2,3]$ developed algorithms to generate drill models by a computer. The drill geometry was analyzed by considering the "slicing" of the drill by arbitrary planes. A computer model was also developed to design a twist drill. Vijayaraghavan and Dornfeld [4] developed an automated drill modeling tool in Solidworks to generate a 3D model of a drill based on the manufacturing parameters of the drill supplied by the user.

Khyratsis et al. [5] developed software routine, which creates the parametrically controlled drill geometries and uses different cutting conditions, achieving the generation of solid models for all the relevant data involved. Nikolcheva et al. [6] developed an automated drill modeling tool in AutoDesk Inventor. The tool uses AutoDesk Inventor to generate a 3D model of a drill based on the manufacturing parameters of the drill supplied by the user.

Kang and Armarego [7, 8] performed a computer-aided "forward" analysis based on a typical automated flute grinder used in drill production to study the flute generation process and the design flute profile of twist drills. They also performed a computer-aided "backward" analysis based on the contact curve method to determine the required grinding wheel profile for generating a given drill flute profile design and the fluting machine settings.

Zhu [9] developed an automated drill modeling tool in Catia to generate a machining feature based on a 3D model of a drill. Based on the contact curve method, he also determined the required grinding wheel profile for a five-axis grinding machine in order to generate a given drill flute profile design.

This paper deals with a CAD (computer aided design) based approach for the grinding wheel design needed in a drill flute production. MATLAB is used to develop a $\mathrm{CAD}$ module for a grinding wheel design which is based on Galloway's [1] and Kang and Armarego's [7, 8] basic framework. The paper involves the following sections: methods, results and conclusions.

\section{Methods}

2.1 The Establishment of the Coordinate Systems and the Machine Setting Variables

In order to model the design flute profile, the drill helical flute surfaces and the grinding wheel profile the coordinate systems have been established as shown in Fig. 2 [8].

The drill helical flute system $O_{1}-X_{1} Y_{1} Z_{1}$ is set to be a moving coordinate system and the wheel coordinate system $\mathrm{O}_{2}-\mathrm{X}_{2} Y_{2} Z_{2}$ to be a fixed system.

The flute grinder involves three machine setting variables, namely, the wheel setting angle $\alpha$ with
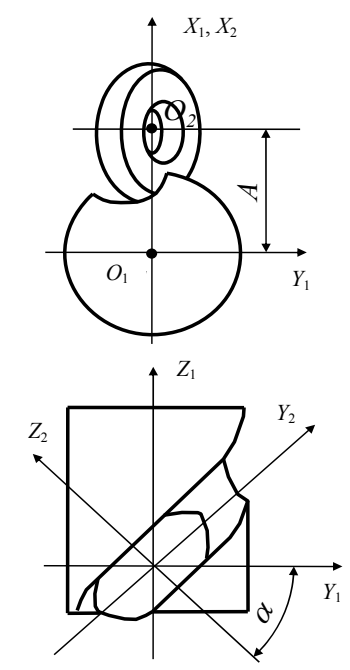

Fig. 2 The established coordinate systems. 
respect to $Y_{1}$, the wheel offset $W O$ along $Z_{2}$, and the vertical distance between the axes of the helical flute and the grinding wheel $A \approx W+R_{g}\left(R_{g}\right.$ is maximum wheel radius).

\subsection{The Modeling of the Design Flute Profile}

The cross-section of the drill has to be designed such that it generates a straight cutting edge when the flanks are ground. The drill flute profile can be divided into six sections, as shown in Fig. 3. Sections 1 and 2 are the unground parts of the drill blank being arcs which make a circle. Sections 3 and 4 are the lip portions of the drill flute profile and Sections 5 and 6 are the heal portions of the drill flute profile.

When the design flute profile is given as a continuous curve, the lip portion of the drill flute profile is obtained from the theoretical profile for a straight lip production and the heel portion profile is represented by a parabolic curve. In this case, explicit mathematical expression for the slopes of the drill profile can then be obtained for any point on the flute surface.

When the lip flute profile is given in a continuous curve for straight lip production, it can be expressed as [8]:

$$
\begin{aligned}
& x=W \cdot \cos \rho+\sqrt{r^{2}-W^{2}} \cdot \sin \rho \\
& y=-W \cdot \sin \rho+\sqrt{r^{2}-W^{2}} \cdot \cos \rho
\end{aligned}
$$

where, $r$ ranges from a half the web thickness $W$ to drill radius $R$ and:

$$
\rho=-\frac{\tan \delta}{R \cdot \tan P} \cdot \sqrt{r^{2}-W^{2}}
$$

The slope of the lip portion of the drill profile is expressed as follows:

$$
\begin{aligned}
& \frac{d x}{d y}= \\
& \frac{\left(w \cdot \sin \rho-\sqrt{r^{2}-w^{2}} \cdot \cos \rho\right) \cdot \frac{\tan \delta}{R \cdot \tan p}+\sin \rho}{\left(w \cdot \cos \rho+\sqrt{r^{2}-w^{2}} \cdot \sin \rho\right) \cdot \frac{\tan \delta}{R \cdot \tan p}+\cos \rho}
\end{aligned}
$$

According to the theoretical lip flute profile design for a straight lip production, it can be shown that the lip

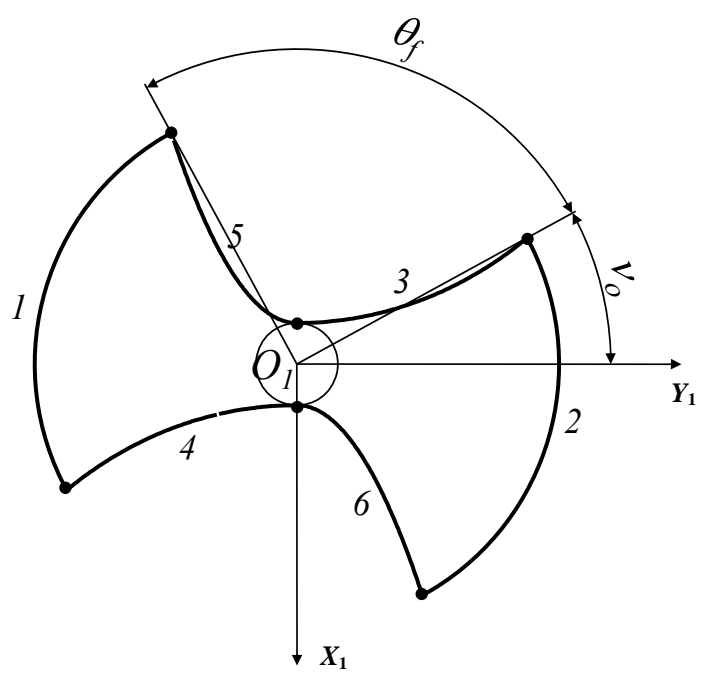

Fig. 3 The drill flute profile.

outer corner of the drill flute profile in the plane normal to the drill axis can be determined from the given drill geometrical specification as follows [1]:

$$
v_{o}=\sin ^{-1}\left(\frac{W}{R}\right)+\frac{\sqrt{R^{2}-W^{2}} \cdot \tan \delta}{R \cdot \tan P}
$$

Therefore, the orientation of the flute profile in the predetermined coordinate system is fixed, i.e., the initial orientation angle $v_{0}$ can be obtained in Eq. (4) based on the theoretical lip flute model which assumes the straight lip is parallel to the $Y_{1}$ axis after the point sharpening as shown in Fig. 1.

The heel flute profile can be substituted by a parabolic curve which passes through the heel corner and tangential to the web thickness or a core passing through the heel corner while the flute subtended angle $\theta_{f}$ is approximately $90^{\circ}$ according to the Chinese standard GB 6137 [10] as shown in Fig. 3. Such a parabolic curve can be expressed as follows:

$$
x=\frac{R \cdot \cos v_{o}-W}{\left(R \cdot \sin v_{o}\right)^{2}} \cdot y^{2}+W
$$

The slope of the heel portion of the drill profile is expressed as follows:

$$
\frac{\mathrm{d} x}{\mathrm{~d} y}=2 \cdot \frac{R \cdot \cos v_{o}-W}{\left(R \cdot \sin v_{o}\right)^{2}} \cdot y
$$




\subsection{Helical Flute Surface Equations}

The helical flute surface can be modelled by adding the flute profile a helical motion along the drill axis, so that the drill helical flute surface can be mathematically represented in a matrix form as follows:

$$
\overrightarrow{r_{1}}=\left\{\begin{array}{c}
X_{1} \\
Y_{1} \\
Z_{1} \\
1
\end{array}\right\}=\left[\begin{array}{cccc}
\cos \phi & -\sin \phi & 0 & 0 \\
\sin \phi & \cos \phi & 0 & 0 \\
0 & 0 & 1 & \frac{R \cdot \phi}{\tan \delta} \\
0 & 0 & 0 & 1
\end{array}\right] \cdot\left\{\begin{array}{c}
x \\
y \\
z \\
1
\end{array}\right\}
$$

where, $\phi$ is an auxiliary variable which represents the rotation of the drill flute profile around the $Z_{1}$ axis to form the helical flute surface, and viewing in the direction along the $Z_{1}$ axis, $\phi$ is positive in clockwise direction. The helical flute surface Eq. (7) with respect to the drill blank axes $O_{1}-X_{1} Y_{1} Z_{1}$ transformed to the wheel coordinate system $\mathrm{O}_{2}-X_{2} Y_{2} Z_{2}$ is expressed as follows:

$$
\overrightarrow{r_{2}}=\left\{\begin{array}{l}
X_{2} \\
Y_{2} \\
Z_{2}
\end{array}\right\}=\left[\begin{array}{ccc}
1 & 0 & 0 \\
0 & \sin \alpha & \cos \alpha \\
0 & -\cos \alpha & \sin \alpha
\end{array}\right] \cdot\left\{\begin{array}{c}
X_{1}-A \\
Y_{1} \\
Z_{1}
\end{array}\right\}
$$

\subsection{The Contact Curve between a Helical Flute Surface and Wheel Surface}

The contact curve between a helical flute surface and a wheel surface can be found in the following vectorial equation [8]:

$$
\overrightarrow{n_{2}} \cdot\left(\overrightarrow{k_{2}} \times \overrightarrow{r_{2}}\right)=0
$$

where, $\overrightarrow{k_{2}}=\left\{\begin{array}{llll}0 & 0 & 1\end{array}\right\}^{\mathrm{T}}$ is the unit vector of $Z_{2}$ axis, $\overrightarrow{n_{2}}$ is the common normal vector of both helical flute surface and wheel surface at each contact point in the $\mathrm{O}_{2}-X_{2} Y_{2} Z_{2}$ system which can be expressed as follows:

$$
\overrightarrow{n_{2}}=\left\{\begin{array}{l}
n_{X_{2}} \\
n_{Y_{2}} \\
n_{Z_{2}}
\end{array}\right\}=\left[\begin{array}{ccc}
1 & 0 & 0 \\
0 & \sin \alpha & \cos \alpha \\
0 & -\cos \alpha & \sin \alpha
\end{array}\right] \cdot\left\{\begin{array}{l}
n_{X_{1}} \\
n_{Y_{1}} \\
n_{Z_{1}}
\end{array}\right\}
$$

where, $\overrightarrow{n_{1}}$ is the common normal vector of both helical flute surface and wheel surface at each contact point in the $O_{1}-X_{1} Y_{1} Z_{1}$ system. The normal vector $\overrightarrow{n_{1}}$ can be expressed as follows:

$$
\overrightarrow{n_{1}}=\left\{\begin{array}{lll}
n_{X_{1}} & n_{Y_{1}} & n_{Z_{1}}
\end{array}\right\}^{T}=\frac{\partial \overrightarrow{r_{1}}}{\partial \phi} \times \frac{\partial \overrightarrow{r_{1}}}{\partial y}
$$

where,

$$
\begin{gathered}
\frac{\partial \overrightarrow{r_{1}}}{\partial \phi}=\left\{\begin{array}{c}
-x \cdot \sin \phi-y \cdot \cos \phi \\
x \cdot \cos \phi-y \cdot \sin \phi \\
\frac{R}{\tan \delta}
\end{array}\right\} \\
\frac{\partial \overrightarrow{r_{1}}}{\partial y}=\left\{\begin{array}{c}
\frac{d x}{d y} \cdot \cos \phi-\sin \phi \\
\frac{d x}{d y} \cdot \sin \phi+\cos \phi \\
0
\end{array}\right\}
\end{gathered}
$$

By substituting Eqs. (8) and (10) into Eq. (9), the contact curve equation can be expressed in an analytical form as follows:

$$
\begin{aligned}
& \overrightarrow{n_{2}} \cdot\left(\overrightarrow{k_{2}} \times \overrightarrow{r_{2}}\right)=\frac{R}{\tan \delta} \cdot\left(\frac{d x}{d y} \cdot \sin \phi+\cos \phi\right) . \\
& {\left[(x \cdot \sin \phi+y \cdot \cos \phi) \cdot \sin \alpha+\frac{R \cdot \phi \cdot \cos \alpha}{\tan \delta}\right]+} \\
& {\left[\begin{array}{l}
\frac{R}{\tan \delta} \cdot\left(\frac{d x}{d y} \cdot \cos \phi-\sin \phi\right) \cdot \sin \alpha- \\
\left(x \cdot \frac{d x}{d y}+y\right) \cdot \cos \alpha
\end{array}\right] .} \\
& (x \cdot \cos \phi-y \cdot \sin \phi-A)=0
\end{aligned}
$$

In order to determine the contact curve in descrete form points $(x, y)$ and corresponding slopes $\mathrm{d} x / \mathrm{d} y$ from design flute profile shown in Fig. 3, from the lip outer corner to the heel outer corner, machine setings variables $\alpha$ and $A$ have to be substituted in Eq. (13). The corresponding value of $\phi$ can be found in solving this equation. The coordinates of the point on the contact curve corresponding to $(x, y, \phi)$ on both the helical flute surface and the wheel surface in $\mathrm{O}_{2}-X_{2} Y_{2} Z_{2}$ system can be found in Eq. (7).

\subsection{The Determination of the Wheel Profile}

When the contact curve is obtained, the grinding wheel surface can be generated by rotating the contact 
curve about the wheel axis. The wheel profile in the plane containing the wheel axis can be expressed as follows [8]:

$$
\begin{aligned}
& x_{a}=\sqrt{X_{2}^{2}+Y_{2}^{2}} \\
& z_{a}=Z_{2}
\end{aligned}
$$

For the automated flute grinder used in this investigation, the obtained discrete wheel profile would be curve-fitted by two circular arcs.

\subsection{The Determination of the Wheel Offset}

The wheel offset $W O$ is obtained from the theoretical wheel profile as shown in Fig. 4.

The theoretical lip and heal portions of the wheel profile intersect in the point of discontinuity $M$. It is apparent that such as profile is not physically feasible thence that it is not possible to precisely generate the corresponding design flute profile in its entirety. By contrast, a portion of the required wheel profile represented by the curves $A M B$ with a discontinuity at $M$ can be physically achieved. It should be noted that the wheel diameter at the point of discontinuity $M$ also represents the maximum wheel diameter $D g$. Furthermore, the location of the discontinuity along the wheel axis represents the wheel offset $W O$.

\section{Results}

Based on Galloway's [1] and Kang and Armarego's $[7,8]$ basic framework introduced in previous section of this paper, a CAD module for grinding wheel design is developed using MATLAB. The GUI (graphical user interface) of developed CAD module is shown in Fig. 5 .

The input parameters such as drill diameter, helix angle, drill point angle, web thickness, the wheel setting angle, and vertical distance supplied in the GUI, are used to generate the wheel profile. GUI also involves output parameters such as the coordinates of centers and radii of circular arcs which approximate the lip and heal portion of the wheel profile, as well as the widths of lip and heal portion of the wheel profile and the wheel offset shown in Fig. 6, which are obtained for the input parameters supplied by GUI.

The GUI also allows the user to save the obtained discrete wheel profile, parameters of circular arcs

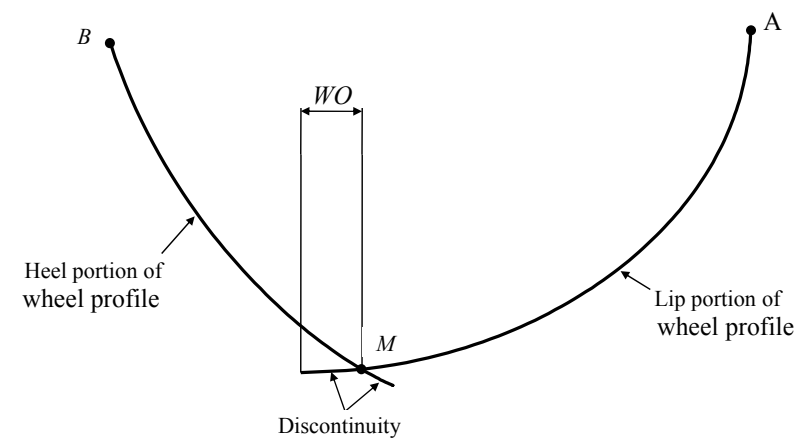

Fig. 4 Theoretical wheel profile.

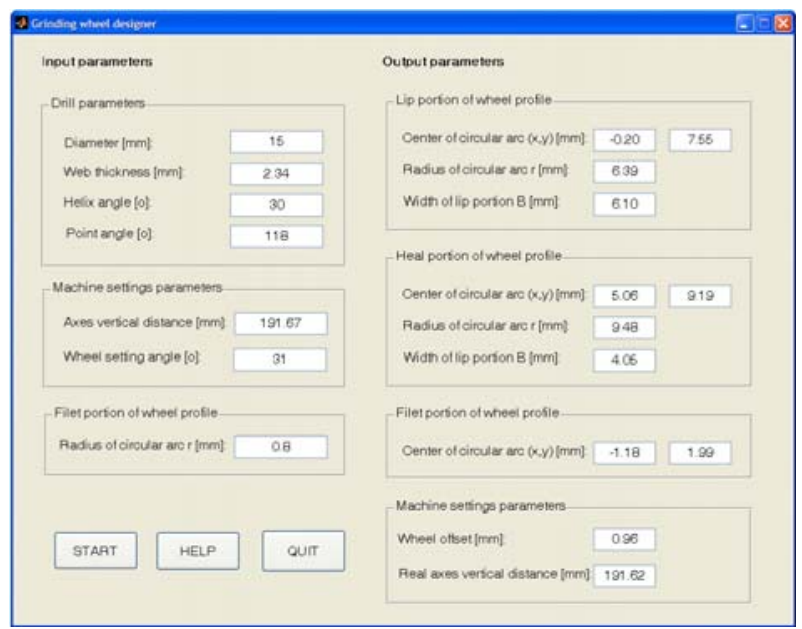

Fig. 5 GUI for automated grinding wheel designer.

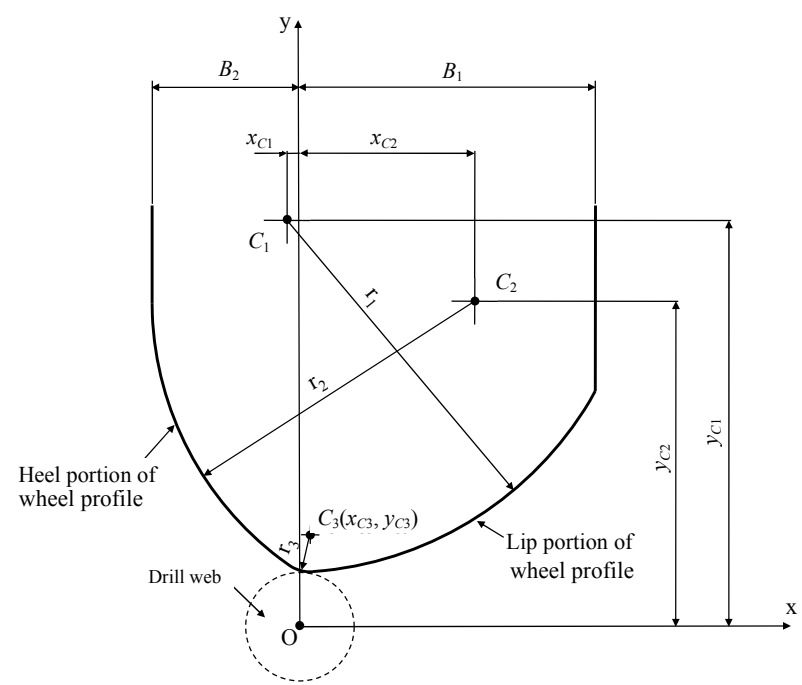

Fig. 6 The wheel profile with parameters of circular arcs approximating its lip and heal portions. 
approximating its lip and heal portions, drill parameters, and machine settings variables in output ASCII files.

The complexity of the flute cutting operation is usually attributed to the phenomenon known as an undercutting. The undercutting manifests itself in the apparent unintended removal of material from the sides of the flutes which necessitates the use of tools whose width is smaller than the width of the desired flute. The reduction of the unintended effects of undercutting can be achieved by changing the machine setting variables. The developed CAD module can be used as a very effective tool for the generation of the wheel profiles and corresponding flute profiles for different machine setup and choosing optimal one regarding the undercutting.

The wheel profiles shown in Fig. 7 are generated for the following set of input parameters:

$D=15 \mathrm{~mm}, 2 p=118^{\circ}, \sigma=30^{\circ}, 2 W=2.34 \mathrm{~mm}$, $A=191.67 \mathrm{~mm}$ and $\alpha=(30 \div 35)^{\circ}$.

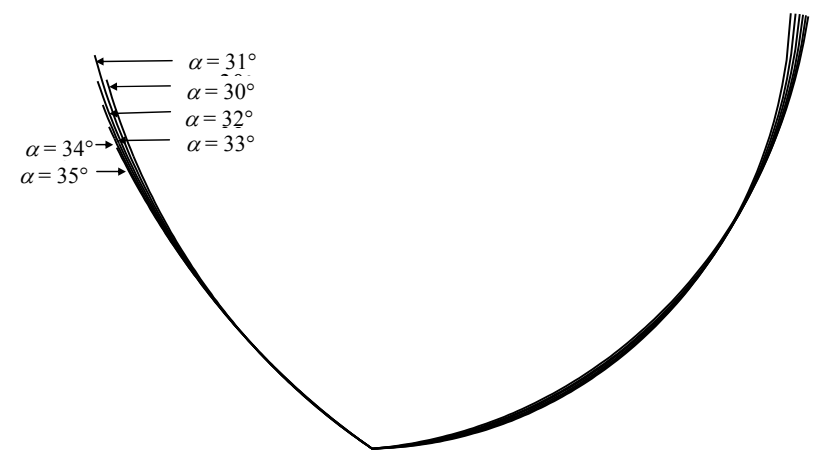

Fig. 7 The wheel profile for different values of the wheel setting angle $\alpha$.

Table 1 Parameters of the wheel profile for different values of the wheel setting angle.

\begin{tabular}{lllllll}
\hline$\alpha(\mathrm{o})$ & 30 & 31 & 32 & 33 & 34 & 35 \\
\hline$x_{C 1}(\mathrm{~mm})$ & -0.12 & -0.20 & -0.28 & -0.38 & -0.45 & -0.57 \\
$y_{C 1}(\mathrm{~mm})$ & 7.39 & 7.55 & 7.70 & 7.86 & 6.82 & 6.99 \\
$r_{1}(\mathrm{~mm})$ & 6.23 & 6.39 & 6.54 & 6.70 & 6.84 & 7.01 \\
$x_{C 2}(\mathrm{~mm})$ & 4.81 & 5.06 & 5.35 & 5.63 & 5.79 & 6.08 \\
$y_{C 2}(\mathrm{~mm})$ & 8.87 & 9.19 & 9.54 & 9.86 & 10.09 & 10.41 \\
$r_{2}(\mathrm{~mm})$ & 9.08 & 9.48 & 9.94 & 10.36 & 10.64 & 11.06 \\
$B_{1}(\mathrm{~mm})$ & 6.10 & 6.17 & 6.23 & 6.28 & 6.32 & 6.36 \\
$B_{2}(\mathrm{~mm})$ & 3.87 & 4.05 & 4.00 & 3.93 & 3.84 & 3.72 \\
$W O(\mathrm{~mm})$ & 0.86 & 0.96 & 1.07 & 1.18 & 1.30 & 1.41 \\
\hline
\end{tabular}

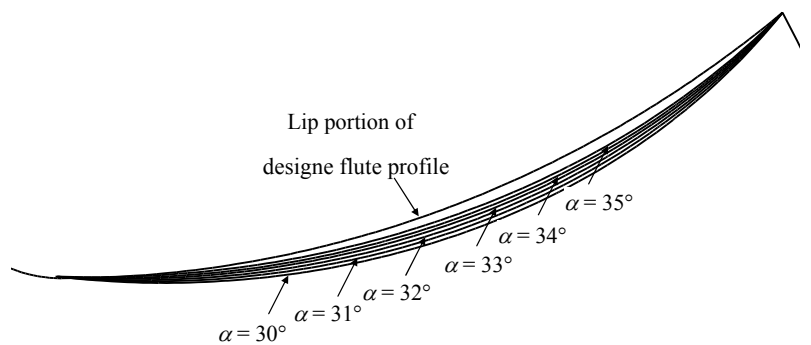

Fig. 8 Lip portion of flute profile for different values of the wheel setting angle $\alpha$.

The wheel profile parameters for different values of the wheel setting angle are given in Table 1. The lip portions of the achieved flute profiles for different values of the wheel setting angle are shown in Fig. 8.

Based on these results, user of this CAD module can simply choose an optimal machine setup giving the best approximation of design flute profile. The flute profiles obtained for the different values of the wheel setting angle, shown in Fig. 8, reveal that the increase of a wheel setting angle results in better approximation of lip portion of design flute profile for the straight lip production.

\section{Conclusions}

The developed automated grinding wheel designer simplifies the design of the grinding wheels for twisted drill flute production and reduces the time for choosing the optimal grinding wheel geometry. Since the complexity of the flute cutting operation is usually attributed to the phenomenon known as an undercutting, this CAD module also can be used as a very effective tool for the generation of the wheel profiles and corresponding flute profiles for different machine setup and choosing optimal one regarding the undercutting. An example of such an approach with the flute profiles obtained for different values of the wheel setting angle is shown in Fig. 8. The user of this CAD module can also modify the other machine setting variables in order to achieve the best approximation of the design flute profile.

\section{References}

[1] Galloway, D. F. 1957. "Some Experiments on the 
Influence of Various Factors on Drill Performance." Transactions of ASME 79: 191-230.

[2] Fuji, S., and DeVries, M. F. 1970. "An Analysis of Drill Geometry for Optimum Drill Design by Computer, Part I-Drill Geometry Analysis." Journal of Engineering for Industry 92: 647-56.

[3] Fuji, S., and DeVries, M. F. 1970. "An Analysis of Drill Geometry for Optimum Drill Design by Computer, Part II-Computer Aided Design.” Journal of Engineering for Industry 92: 657-66.

[4] Vijayaraghavan, A., and Dornfeld, D. 2007. "Automated Drill Modeling for Drilling Process Simulation." Transactions of ASME: Journal of Computing and Information Science in Engineering 7 (3): 276-82.

[5] Khyratsis, P., Bilalis, N., and Antonidis, A. 2009. "CAD Based Predictive Models of the Undeformed Chip Geometry in Drilling." World Academy of Science_Engineering and Technology 52: 318-24.
[6] Nikolcheva, G., Likov, I., and Stoyanov, K. 2012. "Computer Modeling of Twist Drill Shapened at Conical Surface." Machines, Technologies and Materials 12: 62-6.

[7] Kang, D., and Armarego, E. J. A. 2003. "Computer Aided Geometrical Analysis of the Fluting Operation for Twist Drill Design and Production. I Forward Analysis and Generated Flute Profile." Machining Science and Technology 7 (2): 221-48.

[8] Kang, D., and Armarego, E. J. A. 2003. "Computer Aided Geometrical Analysis of the Fluting Operation for Twist Drill Design and Production. II Backward Analysis, Wheel Profile and Simulation Studies." Machining Science and Technology 7 (2): 249-66.

[9] Zhu, J. 2011. "Machining Featured Based Geometric Modeling of Twist Drills." Master thesis, Concordia University.

[10] Chinese standard GB 6137, 1985. 